\title{
人体肝癌体外细胞株 (BEL-7402) 的建立及其特征
}

\author{
陈瑞铭 朱德厚 叶秀珍 沈鼎武 \\ (上海实验生物研究所)
}

肝癌是恶性肿瘤中发病率较高的一种, 它严重地威胁着人民的生命健康. 近年来, 由于新技术的不断发展, 大大提高了对原发 性肝细胞癌的临床诊断率, 但对其防治工作 仍是当前亟待解决的问题.

关于肝癌防治的实验研究, 据许多资料 报道, 绝大多数是采用致癌物质所诱发的动 物肝癌, 包括它的移植的实体型和腹水型或 体外培养建立的细胞株. 这种肝癌的特性与 人体原发性肝癌所具有的特性可能差别甚 大. 因此, 为了满足对人体肝癌的研究需要, 赋予组织培养工作者的任务, 首先是把临床 外科手术切除的肝癌标本, 培养在玻璃瓶内 成为能定期地进行传代生长和性质近似于人 体肝癌的细胞株.

1960 年, 我们培养成功一例人体肝癌细 胞株(代号为 BEL-16), 到目前已历时 14 年. 该株细胞虽然仍能迅速增殖, 但其特性可能 已与原始的特性有所不同. 1974 年初, 我们 又重新培养几例人体肝癌标本, 其中一例已 连续培养 7 个半月. 我们把这株细胞定名为 BEL-7402, 现将这株细胞的培养建株过程及. 其一些特征,简要介绍如下.

我们于 1974 年 2 月 19 日从上海市中山 医院外科手术切除肝癌患者 (男性, 53 岁) 获 得标本. 手术前甲胎蛋白 (AFP) 测定为阳性. 病理学诊断为肝细胞癌, 伴有结节性肝硬化. 在获得标本后两小时内即将癌组织切成小块 进行旋转管法培养, 培养液用含有 $40 \%$ 小 牛血清的 Eagle 氏培养液和适量的抗菌素, $\mathrm{pH} 7.4$, 培养温度是 $37^{\circ} \mathrm{C}$. 经过培养 12 天后,
管壁上的组织小块周围所出现的零散的圆形 细胞、红血球以及细胞碎片就被新长出的多 边形细胞所取代. 新长的细胞长势昰盛, 持 续一个月,后由于管壁上的鸡血浆液化, 影响 了生长速度. 继续培养三个月后, 组织小块 周围的细胞形态仍以多边形的为主, 又逐渐 增快生长率。培养四个月后, 用滴管把管壁 上生长旺盛的细胞群落冲击成为细胞悬液, 接种 2 毫升悬液于小玻璃瓶内, 静置培养(第 一代). 到目前为止, 在玻璃瓶内长成单层的 细胞可每隔 6-7 天传代一次, 已传了 19 代, 历时 7 个半月. 细胞的增长倍数, 如以接种 悬液中的细胞浓度为每毫升 2.5 万个, 则在八 天内可增长 20 倍.

观察和分析单层细胞的活体和染色标 本，除了一些处于有丝分裂阶段和衰老退化 而变圆的细胞不计在内之外, 按细胞形态 分类有三种不同的细胞类型，如表 1 和图 1 所示. 其中以多边形细胞的生长占优势 $(95.9 \%)$. 此外, 尚有少数的梭形细胞和单核 与多核的巨大细胞.

表 1 BEL-7402 细胞的形态分类

\begin{tabular}{c|c}
\hline 细 胞 类 型 & 百分数 (\%) \\
\hline 多边 形 细 胞 & 95.9 \\
梭 形 细 胞 & 1.8 \\
巨大 细 胞 & 2.3 \\
\hline
\end{tabular}

细胞的有丝分裂指数, 根据传代后培养 8 天过程中逐日取样,每次计数 2,000 个细胞 的分裂相,结果表明, 传代后细胞分裂指数逐

本文 1974 年 11 月 4 日收到。 

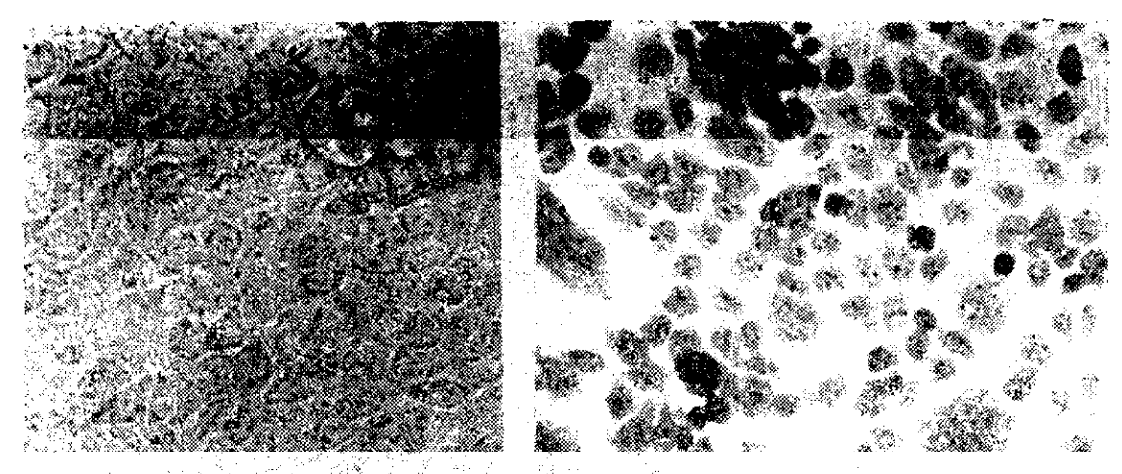

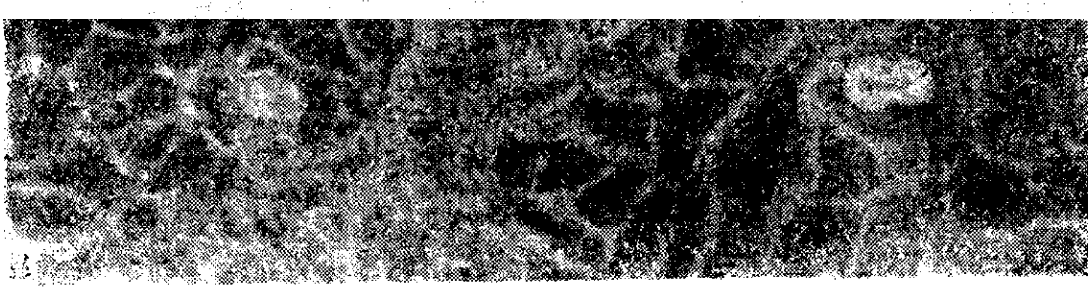

图 1 BEL-7402 细胞单层培新的形态

如图 2 所示. 染色体数的主 流范围在 60-63 个, 占总数 $46.6 \%$,第一主流数为 60 个, 占总数 $15.5 \%$. 在分析中, 发现有一个长而大的近端着 丝点染色体, 其出现频率达 $98 \%$, 为本株细胞的标记染 色体, 如图 3 所示.

接种本株细胞悬液于经 $\mathrm{x}$ 光全身炤射和强的松注射 的刚断乳的雄性大白鼠腋窝 皮下，细胞数量只要 $2.5 \times$ $10^{6}$ 个, 就能在 3-6 天后摸 到皮下有赤豆大的肿瘤结 节; 随着时间的延长, 瘜块 也增大,到 10 天时剖取瘤块 的重量有 80 毫克; 接种细胞 数为 $10 \times 10^{6}$ 个时, 瘤块的 重量为 250 毫克. 㿔块的组 织学观察显示为移植性恶性 肿㿔, 形态特征类似肝细胞 癌, 如图 4 所示.

采用免度荧光法, 即用 荧光素标记的羊抗兔 $\gamma$-球 蛋白抗体与经抗人 AFP 抗 血清处理的单层培养 BEL7402 细胞温浴, 在苂光显微 镜下观察, 初步显示细胞的 荧光强度一般, 而在核膜上 的苂光很强，表明这株细胞

渐上升, 到第四天达最高峰 (占 $69.5 \%$ ), 培 养五天后分裂指数就逐渐下降, 到第 7-8 天 时, 平均值为 $10 \%$ 左右. 分裂的细胞, 以中 期的分裂指数高于其它各期. 异常分裂出现 的频率很高, 所占的比例在培养四天时已超 过正常的分裂指数, 并持续到培养的第八 天.

通过统计和分析 103 个中期分裂相, 得 出 BEL-7402 细胞的染色体数目及其分布,
在体外培养条件下仍能继续产生 AFP. 至于 这株细胞的乳酸脱氢酶 (LDH)、葡萄糖-6磷酸脱氢酶 (G6PD) 和酪氨酸转氨酶 (TAT) 的反应与我们现有的少数几例临床人体肝癌 的酶反应近似，而与人体宿主肝细胞的酶反 应有别, 如 G6PD 酶活力比宿主肝细胞高, 而 $T A T$ 嘸活力则比宿主肝细胞低. 此外, 这株 细胞的超微结构, 就其细胞核、线粒体和内质 网系三者的形状、大小、数量等特点, 也显示 


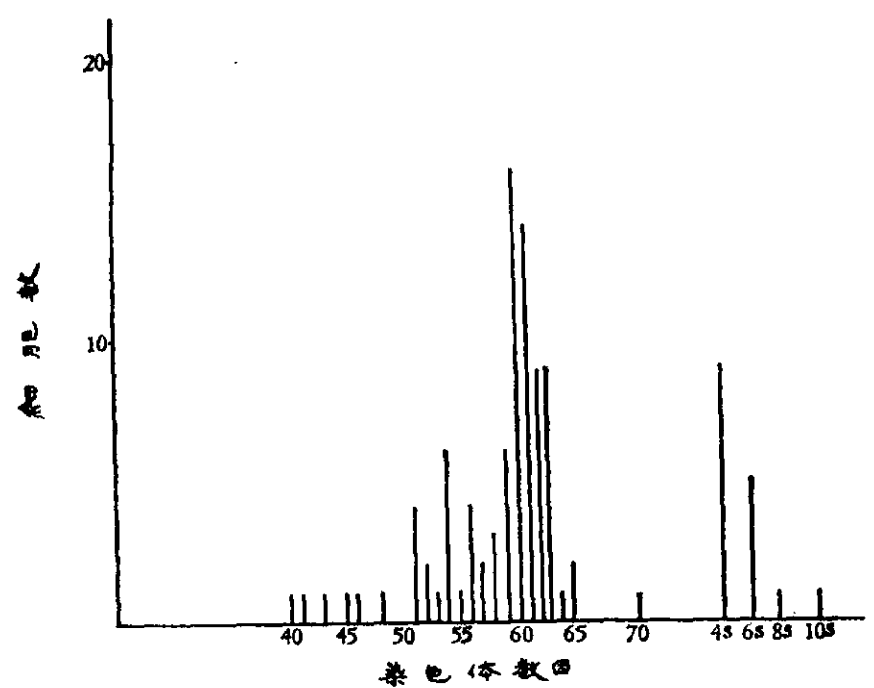

图 2 BEL-7402 细胞的染色体数目分布

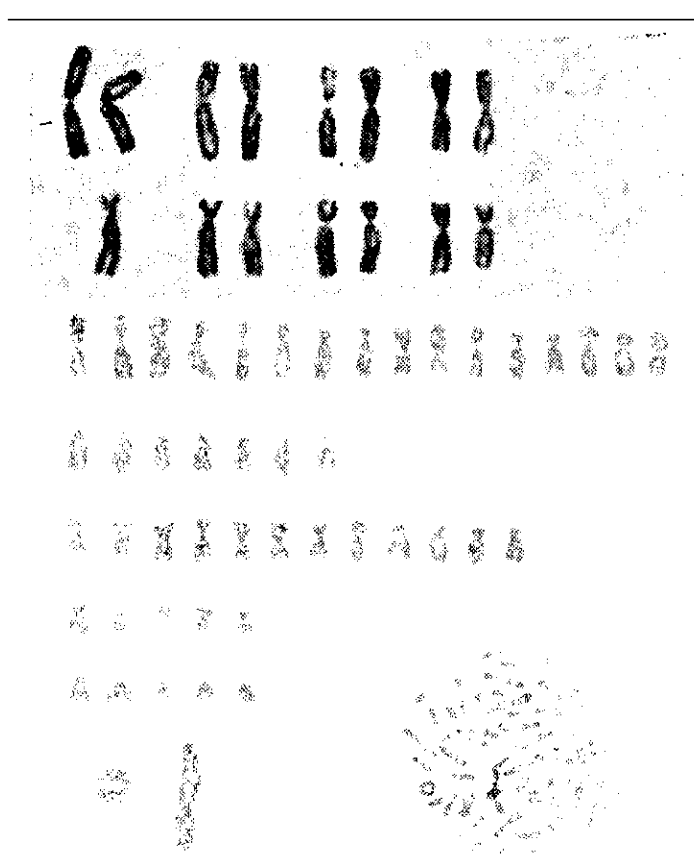

图 3 BEL-7402 细胞的 62 个染色体组型, 示出 1 个长而大的近端着丝点的标记染色 体和 1 个畸变的环化染色体

与临床人体肝痹近似, 而与人正常肝不同.

对这株细胞所作的观察和分析, 归纳为 以下几点:

1. 细胞的增长率既迅速又恒定, 细胞形 态是以多边形上皮样细胞占绝大多数, 正常

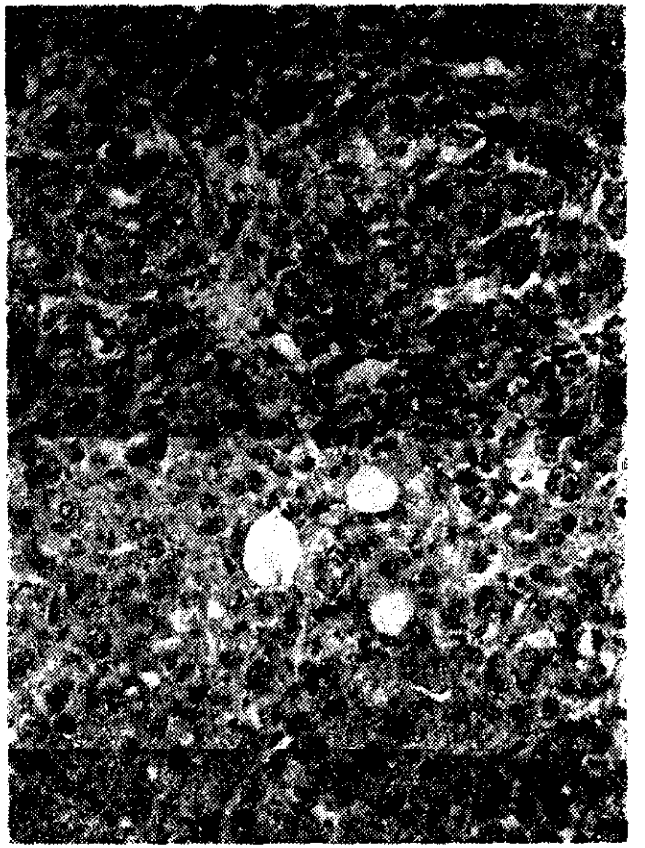

图 4 异种接种 BEL-7402 细胞 $2.5 \times 10^{\circ}$ 个, 10 天后的肿瘤结节切片; Bouin 固定， 苏木精一曙红染色， $\times 184$

和异常的有丝分裂指数均以中期高于其它各 期.

2. 值得引人注意的是:（1）细胞的染色 体主流数为 60 个, 绝大多数细胞有一个近 端着丝点的大型标记染色体；（2）细胞的异 种接种长瘤率高, 瘤块的组织学特点与临床 肝细胞癌相近似;（3）细胞的 AFP 阳性,三 种酶 (LDH，G6PD，TAT) 反应以及超微结 构 (细胞核、线粒体、内质网系)都基本上保持 临床人体肝癌的特征.

我们认为, 这株体外建成的 BEL-7402 细胞, 不仅有助于阐明人体肝癌细胞的生物 学特性, 而且也可能为肝癌的实验治疗以及 其它基础研究提供了一个较为理想的实验模 型.

AFP 的荧光抗体工作由邓正文、姚曾序两同志 协助测定, 特此致谢. 